\title{
Un verre de pipi tous les matins éloigne le médecin. Pratiques informationnelles des internautes en lien avec l'urinothérapie
}

\section{INTRODUCTION}

L'utilisation de l'urinothérapie par un patient, $\mathrm{vu}$ en consultation d'allergologie, nous a conduit à évaluer comment les patients s'informent sur cette pratique. L'essor de l'information de santé grand public favorise la propagation des contenus sur la médecine non conventionnelle. Celle-ci repose sur des croyances et non sur des preuves scientifiques, et n'est pas sans danger.

\section{MATÉRIEL ET MÉTHODES}

Nous avons procédé à la recherche et à l'analyse qualitative de contenu de messages portant sur l'urinothérapie, publiés sur un forum de santé. La constitution et l'analyse du corpus ont été réalisées par un allergologue et un chercheur en sciences de l'information et de la communication.

\section{RÉSULTATS}

Le corpus analysé se monte à 177 messages, publiés entre 2003 et 2017. Les internautes ont recours à l'urinothérapie à la suite d'un échec de thérapie médicamenteuse, accompagné par un manque de confiance en la médecine occidentale et son système de santé. Les non-initiés demandent des informations et de la documentation. Les initiés partagent leur expérience (pathologie soignée, cause du recours à cette thérapie, procédure d'usage) et s'appuient sur les sources d'information (sites web, forums, articles scientifiques, blogs, livres, experts), visant à prouver la légitimité de la méthode. Alors qu'une partie des internautes présente les effets bénéfiques de l'urinothérapie, une autre est sceptique et souligne le manque de résultats et la dangerosité. Ces prises de position sont accompagnées par l'empirie, les citations des sources d'information, des messages malpolis et méprisants, et la rationalisation qui imite le discours scientifique.

\section{Exemple : l'un des messages du corpus analysé}

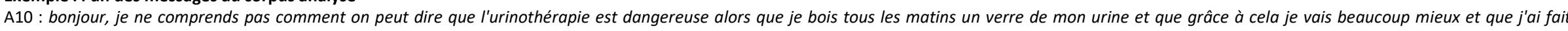

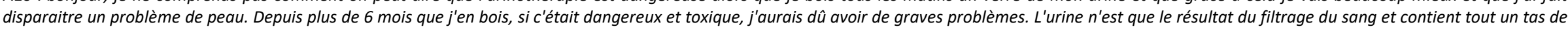

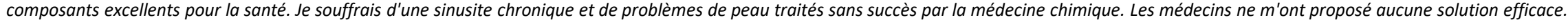

Le recours à l'urinothérapie résulte, en grande partie, d'une crise de confiance dans la médecine occidentale et le secteur de la santé, et la citation des sources d'information et des retours d'expérience dans les échanges d'internautes sont une tentative d'attribuer de la légitimité à cette pratique. II est important de connaître l'existence de ces thérapies alternatives, fréquentes dans le domaine de l'allergologie, et implémenter la discussion scientifique entre médecin et patient.

MOTS CLÉS : URINOTHERAPIE - MEDECINE NON CONVENTIONNELLE - PRATIQUES INFORMATIONNELLES FORUMS DE SANTE GRAND PUBLIC 